\title{
The Effectiveness of Aerobic Exercises at difference Intensities of Managing Blood Pressure in Essential Hypertensive Information Technology Officers
}

\author{
Mohamad Razali Abdullah', Vijayamurugan Eswaramoorthi², Rabiu Muazu Musa', Ahmad Bisyri Husin Musawi Maliki', \\ Norlaila Azura Kosni ${ }^{1}$, Mainul Haque ${ }^{3}$ \\ ${ }^{1}$ Faculty of Applied Social Sciences, Universiti Sultan Zainal Abidin, 21300, Terengganu, Malaysia. \\ ${ }^{2}$ Faculty of Health Sciences, Universiti Sultan Zainal Abidin, 21300, Terengganu, Malaysia. \\ ${ }^{3}$ Unit of Pharmacology, Faculty of Medicine and Defense Health, National Defense University of Malaysia, Kem Sungai Besi, 57000 Kuala Lumpur, Malaysia.
}

\begin{abstract}
Background: The appropriate exercise intensity has been shown to be effective in managing essential hypertension. However, this can be achieved only when an individual is aware of the right volume of the exercise intensity to be undertaken. This study aims to ascertain the effectiveness of aerobic exercises at differing intensities of managing blood pressure in Essential Hypertensive (EHT) Information Technology Officers (ITO). Methods: Forty-five EHT ITO male participants between the age of 35-45 years with a BMI less than $30 \mathrm{Kg} \mathrm{m}^{-2}$ were randomly allotted into three groups (A, B, C) of 15 each. All groups were given four weeks' aerobic interval training of brisk walking for 45 minutes per day for four times a week using a treadmill. The intensity of the exercise was determined and graded based on the Borg scale Rate of Perceived Exertion (RPE), different intensities were assigned to each group; group A low intensity (RPE 11), group B moderate intensity (RPE13), and group C high intensity (RPE15). The pre and post intervention resting blood pressure values of all the three groups were measured and statistically compared. Results: All the three intensities of the aerobic exercises are effective in managing the systolic and diastolic blood pressure in the EHT ITO. However, medium intensity aerobic exercise is found to be highly effective in managing the blood pres-
\end{abstract}

sure in EHT ITO compared to low and high intensities. Conclusion: The current study suggests to the general public working under various sectors of similar nature with the ITO to follow the appropriate intensity exercise revealed from this study.

Key words: Aerobic exercise, Blood pressure, Borg scale, Essential Hypertension, Information Technology Officers.

Correspondence :

Dr. Mainul Haque

Professor, Unit of Pharmacology, Faculty of Medicine and Defense Health, National Defense University of Malaysia, Kem Sungai Besi, 57000 Kuala Lumpur, Malaysia.

Land Line: +60 96275674 (Office), +60 96231319 (Home)

Cell Phone: + 60 10, 926 5543, +60 108076436 .

Fax No: +60 96275639 (Inland), +60 96221707 (Overseas)

E-mail: runurono@gmail.com

DOI: 10.5530/jyp.2016.4.27

\section{INTRODUCTION}

Hypertension is defined randomly at levels above, generally accepted normal. Generally accepted normal blood pressure is less than 120 mmHg systolic and less than $80 \mathrm{mmHg}$ diastolic. ${ }^{1}$ According to etiology, the hypertension is classified into two; Primary or Essential Hypertension and Secondary or Inessential hypertension. ${ }^{1}$ Essential Hypertension: Research has shown that about 85 percent of patients with hypertension are seen under this category. It is not possible to define a specific underlying cause. For example, in 70 percent of people in the family, one of the family members is affected. Essential Hypertension is especially frequent in some races, particularly American Blacks and Japanese. It is common in countries where there is a high salt intake. ${ }^{1}$ However, in Secondary Hypertension: 5-10 percent of people with hypertension are seen under this category. Hypertension that occurs as a consequence of a specific disease or abnormality is classified as secondary hypertension. The causes are Coarctation of the Aorta, Renal disease, Endocrine Disorders, Alcohol, Drugs, and Pregnancy. ${ }^{1,2}$

The pathogenesis of Essential Hypertension is not plainly understood. However, it is known that the underlying defect is an increase in peripheral vascular resistance. Therefore, hypertension is a serious medical problem if left untreated. ${ }^{1}$ A variety of Antihypertensive treatments and drugs are available to control hypertension. Similarly, physical activity has also been an effective treatment. High levels of physical activity and fitness have often been discovered to be indirectly associated with the occurrence and severity of hypertension in epidemiological surveys. ${ }^{3,4}$ Moreover, several recent clinical trials have demonstrated that physical activity reduces blood pressure in hypertensive and normotensive persons irrespective of weight loss. ${ }^{5}$ Consequently, various reviews and the Joint Committee on Detection, Evaluation, and Treatment of high Blood Pressure have concluded that exercise can be beneficial in the management of hypertension..$^{5-7}$ Aerobic exercises are helpful in managing hypertension in order to increase the cardiovascular function and local muscular endurance. ${ }^{8}$ It also reduces the fat mass of the body and plays a vital role in Osteoporosis. ${ }^{9}$

The American College of Sports Medicine (ACSM) describes aerobic exercise as "any activity that uses big muscle groups that can be sustained continuously, and is rhythmic in nature." It is a type of exercise that overloads the heart and lungs and causes them to work harder rather than at rest. ${ }^{10}$ Steady aerobic exercise is broadly recommended for EHT. ${ }^{11}$ Many studies have been done regarding the effectiveness of aerobic exercise on blood pressure. Likewise, numerous studies have shown that aerobic exercise has an antihypertensive effect. ${ }^{12-17}$ Previous studies have indicated that aerobic exercise could be divided into three types depending on the intensity of training namely; low, medium and high intensity. Several studies exist comparing two intensities of aerobic exercise on blood pressure and proved that lower intensity aerobic exercise resulted in greater blood pressure reduction as well as high intensity aerobic exercise. ${ }^{18}$ Meanwhile, some study compares all the three intensities of aerobic exercise on healthy subjects. ${ }^{19}$ However, there are limited studies undertaken to examine the effects of all three intensities of aerobic exercises on the patient population. Nonetheless, research has also shown that adherence to an exercise program is poor, that is within six months of the com- 
mencement of exercise program about 50 percent of individuals had stopped continuing regular exercise. One explanation for poor adherence is the intensity of exercise undertaken, which is initially too high, and it is physically and psychologically daunting for individuals who had previously led sedentary lives. ${ }^{20}$ Therefore, to get to an effective control over blood pressure prescription of the appropriate intensity of aerobic exercise is crucial. Information Technology (IT) industry in India has acquired a remarkable boost due to the globalization of Indian economy and favorable government policies. ${ }^{21}$ IT and IT related specialists are at a constant pressure to deliver services proficiently and at a cost effective rate. Staffs working in these IT industries are susceptible to grow a lot of health problems due to incessant physical and mental stress of their job. One of the serious health concerns that could be encountered by these people is high blood pressure or otherwise EHT. Among the best way to tackle this health issue is through physical exercise due to the available evidence reporting the effectiveness of aerobic exercise in the management of EHT. In response to this problem, some of the developed IT industries in the cities have built gymnasiums in their domain and employed professional exercise scientists to prescribe a proper exercise to their staffs in view to managing their stress and raised blood pressure. However, there is a growing increase in the number of developing IT industries in some part of India such as Bangalore; these industries are seeking for rapid recognition and as such places high demands on their workers to achieve target within a short period. These industries are on their developmental stage and therefore could not provide the facilities and trained personnel for their officers to provide the necessary guidelines and prescriptions for a proper exercise, leaving the officers with no choice than to depend on their knowledge and assumptions. Although, the ITO is working under these industries recognized the value of exercise during their work free hour, and the majority is engaged in the exercise, but the rate at which the exercise is performed based on selfprescribing with no specific guideline or protocol. These problems lead to the sustenance of injuries and development of discomforts. For this reason, the adherence to the exercise by the officers suffered a setback and consequently leads to withdrawal. Hence, preventive strategies are therefore urgently needed to address and manage this prevalent through the prescription of a proper exercise to manage and optimize the rate of hypertension among these ITO. The aim of this study is to examine the effectiveness of low, moderate as well as a high intensity aerobic exercise in managing the blood pressure in EHT ITO and to compare the effectiveness of these three intensities of exercises in managing the blood pressure in EHT ITO.

\section{MATERIALS AND METHODS}

Participants: Forty-five sedentary EHT male participants working in IT industries residing in Bangalore between the age of 35 to 45 years with a BMI less than $30 \mathrm{Kg} / \mathrm{m}^{2}$ and resting blood pressure between 130/85 $\mathrm{mmHg}$ and $159 / 99 \mathrm{mmHg}$ screened by a physician were included in this study. Procedure: The EHT participants for the study were those referred by a physician at the Vinayaka health care clinic. A general assessment chart was used for collecting demographic information for determining inclusion and exclusion criteria; this included measuring Height, Weight, and BMI. Informed consent was obtained from participants who met the criteria. Forty-five participants were selected from the population by using Non-probability convenient sampling method. Sampled participants were randomly assigned into three groups; Group $\mathrm{A}, \mathrm{B}$, and $\mathrm{C}$ with 15 participants in each group. Resting blood pressure of all the participants was measured prior to the commencement of the study by using Omron Blood Pressure Measurement Device. Borg scale was explained to the participants. Participants in all the groups were prescribed aerobic training on the treadmill. The parameters consist of brisk walking on a treadmill in an aerobic interval method for the duration of 45 Minutes per day, four times per week. The aerobic exercise intensity varied for the three groups described as follows: Group A: Participants in this group were given a Low-Intensity Aerobic Exercise with Borg scale RPE at 11. Participants were asked to maintain this intensity of rate of perceived exertion throughout the 45 minutes' duration of the aerobic training. Group B: Participants in this group were given a Moderate Intensity Aerobic Exercise with Borg scale RPE at 13. Participants were asked to maintain this intensity of rate of perceived exertion throughout the 45 minutes' duration of the aerobic training. Group C: Participants in this group were given a High-Intensity Aerobic Exercise with Borg scale RPE at 15. Participants were asked to maintain this intensity of rate of perceived exertion throughout the 45 minutes' duration of the aerobic training. Participants were instructed to do their respective aerobic exercise in the morning between 7.00 am to $9.00 \mathrm{am}$. At the end of the fourth week resting blood pressure was measured and recorded, in spite of periodic blood pressure measurement. The pre and post study resting blood pressure values of three groups were statistically compared. Ethical Consideration: Due to the nature of this study involving human subjects, ethical clearance was obtained from the University with a memo number 04MT645 and all the subjects involved were duly informed about the nature of the study and signed consent form. Statistical Analysis: Paired samples t-test was used to find out the significance differences of Blood Pressure values between the pre and post aerobic training and Analysis of variance (ANOVA) was used to determine the significance difference between the different intensity exercise groups. Moreover, Post hoc test (Tukey's test) was utilized to determine the point of the paired sample after (ANOVA) was found to be significant. All the statistical analysis was conducted using Graph pad prism 4.03 versions and XLSTAT version 2014 add-in software (New York, USA) at a confidence level of $\mathrm{p} \leq 0.05$.

\section{RESULTS}

Table 1 shows the distribution of the mean and standard deviation of the pre and posts aerobic training for both systolic and diastolic blood pressures, respectively, after the intervention carried out for eight weeks on the three groups. It also showed a decrease in average systolic and diastolic readings after the intervention in the three groups. One-way ANOVA from Table 2 shows significant differences in both systolic and diastolic blood pressure in post aerobic training but shows no significant difference in both systolic and diastolic blood pressure in pre-aerobic training. Then, with the existence of a significant difference in both systolic and diastolic blood pressure in post aerobic training, pair wise significance test was analyzed. Table 3 reveals the multiple comparisons for both SBP and DBP between the three groups at the post aerobic training. From the table, it can be seen that for SBP multiple comparisons, group A \& B has a significant improvement, group A \& C have no significant improvement while group B \& C have a highly significant improvement. However, for the DBP multiple comparisons, group A \& B have shown a significant improvement, group A \& C has no significant improvement whereas group B \& C have a highly significant improvement. Figure 1 reflects a box and whisker plots for the means comparisons at the post aerobic training phase of the three group's different intensities on both SBP and DBP. It can be seen from the plots that the means of the moderate intensity exercise group are significantly lower as compared to the other groups of the low and high intensity in both SBP and DBP of post training program.

\section{DISCUSSION}

The purpose of the current study is to determine the effectiveness of aerobic exercises at differing intensities of managing blood pressure in EHT ITO. To achieve the purpose of this study ITO, who were initially 
Table 1: Distribution of mean and standard deviation of pre and post aerobic training for SBP and DBP of participants in the three groups

\begin{tabular}{ccccc}
\hline \multirow{2}{*}{ Group } & \multicolumn{2}{c}{ SBP } & \multicolumn{2}{c}{ DBP } \\
\cline { 2 - 5 } & Pre-training & Post Training & Pre-training & Post Training \\
\hline A & $140.0(4.50)$ & $133.6(3.94)$ & $90.0(4.54)$ & $84.1(3.39)$ \\
B & $140.1(8.31)$ & $127.7(5.90)$ & $89.53(3.82)$ & $81.27(2.49)$ \\
C & $140.2(8.28)$ & $134.5(6.61)$ & $89.67(3.48)$ & $86.67(3.35)$ \\
\hline
\end{tabular}

Table 2: One-way ANOVA scores for the pre and post aerobic training SBP and DBP of participants in the three groups

\begin{tabular}{lcc}
\hline Different groups & Calculated F value & Result \\
\hline & Systolic blood pressure & \\
Pre aerobic training & 0.002953 & $\mathrm{P}>0.05$ \\
Post aerobic training & 6.504 & $\mathrm{P}<0.05$ \\
& Diastolic blood pressure & \\
Pre aerobic training & 0.05506 & $\mathrm{P}>0.05$ \\
Post aerobic training & 11.34 & $\mathrm{P}<0.05$ \\
\hline
\end{tabular}

Table 3: Tukey's multiple comparison tests for the post aerobic training SBP and DBP of participants in the three groups

\begin{tabular}{lcc}
\hline Pairs & P value & Pairwise Significance \\
\hline & Systolic Blood Pressure & \\
Group A \& B & $\mathrm{P}<0.05$ & Significant \\
Group A \& C & $\mathrm{P}>0.05$ & Not Significant \\
Group B \& C & $\mathrm{P}<0.01$ & Highly Significant \\
& Diastolic Blood Pressure & \\
Group A \& B & $\mathrm{P}<0.05$ & Significant \\
Group A \& C & $\mathrm{P}>0.05$ & Not Significant \\
Group B \& C & $\mathrm{P}<0.001$ & Highly Significant \\
\hline
\end{tabular}

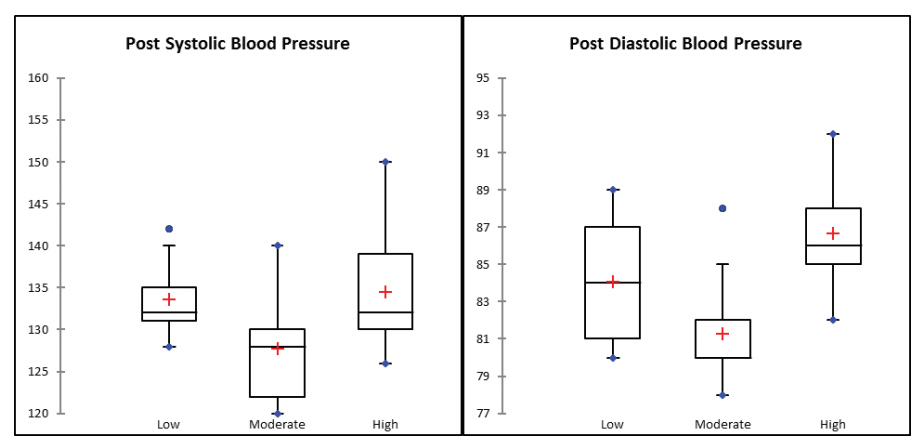

Figure 1: Box and whisker plots of the three groups' difference intensity on post systolic and diastolic blood pressure.

diagnosed with EHT was randomly allotted into three groups $(\mathrm{A}, \mathrm{B}, \mathrm{C})$ of 15 each. All groups were given four weeks' aerobic interval training of brisk walking for 45 minutes per day for four times a week using a treadmill. A Borg scale was used for prescribing the intensity of aerobic exercise which is recommended by the previous researcher. ${ }^{20}$ The results for the comparative mean scores of pre aerobic training SBP of all the three groups reveal no significant difference. But the post aerobic training mean scores of all the three groups are significantly different from each other (see Table 1). The one-way ANOVA for the pre aerobic training SBP of all the three group comparison shows $\mathrm{P}$ value of more than $0.05(\mathrm{P}>0.05)$, which indicate that there is no significant difference between the pre aerobic training participants of the three groups. The one-way ANOVA for post aerobic training SBP of all the three group comparison shows $\mathrm{P}$ value of less than $0.05(\mathrm{P}<0.05)$, which indicate that there is a significant difference between the post aerobic training participants of the three groups. Meanwhile, the comparative mean scores of the pre and post aerobic training DBP of all the three groups show that the pre aerobic training means scores of all the three groups are similar. But the post aerobic training mean scores of all the three groups are different from each other. Likewise, the one-way ANOVA for pre aerobic training DBP of all the three group comparison shows $\mathrm{P}$ value of more than $0.05(\mathrm{P}>0.05)$, which indicate that there is no significant difference between the pre aerobic training participants of the three groups. However, the one-way ANOVA for post aerobic training DBP of all the three group comparison shows $\mathrm{P}$ value of less than $0.05(\mathrm{P}<0.05)$, which indicate that there is a significant difference between the post aerobic training participants of three groups (Table 2). In general, These results show that aerobic exercise at various intensities has significantly different effects in managing the SBP \& DBP in EHT ITO. The results of this study are consistent with the prior studies where they found that the influence of aerobic exercise on blood pressure varies according to the intensity of training. ${ }^{19}$

The Tukey's multiple comparison tests for post SBP and DBP in the three groups also indicate that the medium intensity aerobic exercise is the best to control the SBP \& DBP in EHT ITO as projected in (Table 3 and Figure 1). Finding from this study is parallel with the previous study which found that moderate intensity aerobic exercise is most effective to manage the blood pressure of normal participants, compared to low \& high intensity aerobic exercise. ${ }^{20}$ Nevertheless, some study investigates the effect of moderate intensity aerobic exercise and high intensity aerobic exercise on blood pressure and also another study examines the effect of all the three intensities of aerobic exercise on the High Density Lipoprotein profile. ${ }^{22-24}$ Moreover, a comparative study on the effect of all the three intensities of aerobic exercise on blood pressure in normal participants have been conducted in which revealed that medium aerobic exercise is more effective in managing high blood pressure. ${ }^{20}$ Thus, the finding of this study has also shown that moderate intensity exercise has the best efficacy in managing EHT ITO population.

\section{CONCLUSION}

The daily effect of IT on our lives continues unrestricted. As advancements and computer capacities increase, this influence will continue to grow in the coming years at an increasing rate. As innovation advances, there is additionally expanded stress that is connected with the so-called "technology stress". This brings additional pressure on individuals to adjust to new headways and upgrade their knowledge in their field. This development could come with a price, the health status of such individuals directly involves in the IT industries could be at risk unless the right measure is taken to avert such disaster. To manage stress and the consequent essential hypertension inherent to it, these individuals need to engage in regular exercises at the proper intensity. The findings of the current study indicate that all the three intensities of aerobic exercises are effective in managing the blood pressure in EHT ITO. However, medium intensity aerobic exercise is found to be highly effective to manage the blood pressure in EHT ITO compares to high and low intensities aerobic exercises. It is therefore recommended that ITO should engage in medium aerobic exercise, at least, four days per week and 45 minutes per day for an effective management of their high blood pressure. The finding of the current study could also be useful for the general working population 
in the different sector of establishments of similar nature with the ITO

to adhere to the proper and efficient exercise intensity proposed by this study for the benefit of their health.

\section{ACKNOWLEDGEMENTS}

The authors wish to extend their sincere gratitude to all the participants for their help and cooperation throughout the data collection process.

\section{CONFLICT OF INTEREST}

Authors possess no conflict of interest.

\section{ABBREVIATIONS USED}

EHT: Essential Hypertensive; ITO: Information Technology Officers; RPE: Rate of Perceived Exertion.

\section{ABOUT AUTHORS}

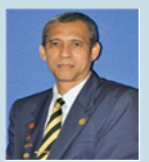

Mohamad Razali Abdullah: Obtained his Bachelor of Physical Education in 1989 from the University Putra of Malaysia, Master of Science in Sport and Exercise Science from University of Wales Institute, Cardiff in 1998 and Doctor Philosophy of Sports Science in 2007 from the University Putra of Malaysia. His interests were motor control, sports biomechanics and motor performance. He is currently working as lecturer at Faculty of Applied Social Sciences, University of Sultan Zainal Abidin, Kuala Terengganu, Malaysia.



VIJAYAM URUGAN ESWARAMOORTHI: Is a Part time doctoral student at University Sultan Zainal Abidin (UniSZA) Malaysia. He obtained his Bachelor of Physiotherapy in 2004 from The Tamil nadu Dr. M.G.R Medical University Chennai, India and graduated in Master of Physiotherapy in Cardiorespiratory and ICU at Rajiv Gandhi University of health sciences Karnataka in 2006. His current research focused on Paralle Diaphragm-Pelvic Breathing on Pulmonary function. His research interest include Cardio-respiratory Physiotherapy and Exercise Science. He is currently working as a lecturer at Faculty of Health sciences, UnisZa Kuala Terengganu, Malaysia.

RABIU MUAZU MUSA: Is a doctoral student at University Sultan Zainal Abidin (UniSZA), Malaysia. He obtained his bachelor degree at Bayero University Kano, Nigeria in Physical and Health Education in 2011 and graduated in Master of Sport Science at UniSZA in 2015. His doctoral research focused on developing a model for athletic performance. His research interests include performance analysis, health promotion, sports psychology, exercise science, talent identification as well as test and measurement.

\section{REFERENCES}

1. De Bano DP, Boon NA. Diseases of the cardiovascular system. In: Edwards CRW and Bouchier IAD, Ed. Davidson's principles and practice of Medicine. $16^{\text {th }}$ Edition. Edinburgh, Scotland. Churchill Livingstone. 1991;318-9.

2. Peter R, Alfredsson L, Hammar N, Siegrist J, Theorell T, Westerholm P. High effort, low reward, and cardiovascular risk factors in employed Swedish men and women: baseline results from the WOLF Study. J Epidemiol Community Health. 1998;52(9):540-7.

3. Montoye HJ, Metzner HL, Keller JB, Johnson BD, Epstein FH. Habitual physical activity and blood pressure. Med Sci Sports. 1972;4(4):175-81.

4. Blair SN, Goodyear NN, Gibbons LW, Cooper KH. Physical fitness and incidence of hypertension in healthy normotensive men and women. JAMA. 1984;252(4):487-90.

5. Leon AS, Blackburn H. 1982. Physical activity and hypertension. In: Freis, Ed Cardiology Hypertension. New York, USA. Butter worth \& Co. 14-36.

6. Scals DR, Hagberg JM. The effect of exercise training on human hypertension: A review. Med Sci Sports Exerc. 1984;16(3):207-15.

7. The 1988 report of the Joint National Committee on detection, evaluation, and treatment of high blood pressure. Arch Internal Medicine. 1988;148:1023-38.

8. Åstrand PO. Physical performance as a function of age. JAMA. 1968;205(11): 729-33.

9. Hatori M, Hasegawa A, Adachi H, Shínozaki A, Hayashi R, Okano H, et al. The effects of walking at the anaerobic threshold level on vertebral bone loss in postmenopausal women. Calcif Tissue Int. 1993;52(6):411-4.

10. The Exercise and Physical Fitness. Georgia: Science program in Exercise Science in the Department of Kinesiology and Health at Georgia State University. Updated 1999 April 29; cited 2016 April 12. Available from: http://www2.gsu. edu/ wwwfit/

11. Martin JE, Dubbert PM, Cushman WC. Controlled trial of aerobic exercise in hypertension. Circulation. 1990;81(5):1560-7.
12. Duncan JJ, Farr JE, Upton SJ, Hagan RD, Oglesby ME, Blair SN. The effect of aerobic exercise on plasma catecholamines and blood pressure in patients with mild essential hypertension. JAMA. 1985;254(18):2609-13.

13. Powers S. 2011. Exercise physiology: Theory and application to fitness and performance. McGraw-Hill Higher Education.

14. Buys R, Avila A, Cornelissen VA. Exercise training improves physical fitness in patients with pulmonary arterial hypertension: A systematic review and metaanalysis of controlled trials. BMC pulmonary medicine. 2015;15(1):1-9.

15. Mersy DJ. Health benefits of aerobic exercise. Postgrad Med. 1991;90(1): 103-7, 110-2

16. Fagard RH. Physical fitness and blood pressure. J Hypertens. 1993;1(11):47-52

17. Kelley G, McClellan P. Antihypertensive effects of aerobic exercise. A brief metaanalytic review of randomized controlled trials. Am J Hypertens. 1994;7(2):115-9.

18. Petrella RJ. How effective is exercise training for the treatment of hypertension? Clin J Sport Med. 1998;8(3):224-31.

19. Kingwell BA, Jennings GL. Effects of walking and other exercise programs upon blood pressure in normal subjects. Med J Australia. 1993;158(4):234-8.

20. Paley CA. A way forward for determining optimal aerobic exercise intensity? Physiotherapy. 1997;83(12):620-4

21. Padma V, Anand NN, Gurukul SS, Javid SS, Prasad A, Arun S. Health problems and stress in Information Technology and Business Process Outsourcing employees. J Pharm Bioallied Sci. 2015;7(Suppl 1):9-13

22. Kokkinos PF, Papademetriou V. Exercise and hypertension. Coron Artery Dis 2000; $11(2): 99-102$

23. Spate-Douglas T, Keyser RE. Exercise intensity: its effect on the high-density lipoprotein profile. Arch Phys Med Rehabil. 1999;80(6):691-5.

24. Sinha S, Akhter QS, Banik S, Islam MZ, Haque M. Correlation Study of Insulin Resistance and Essential Hypertension among Bangladeshi Male Volunteers. J Young Pharm. 2015;7(3):200-5. 